\title{
Generalized Mechanistic Model for the Chemical Vapor Deposition of 2D Transition Metal Dichalco- genide Monolayers
}

Ananth Govind Rajan ${ }^{1}$, Jamie H. Warner ${ }^{2}$, Daniel Blankschtein ${ }^{1}$, and Michael S. Strano ${ }^{1}$ *

${ }^{1}$ Department of Chemical Engineering, Massachusetts Institute of Technology, Cambridge, Massachusetts 02139, United States

2 Department of Materials, University of Oxford, Parks Road, Oxford OX1 3PH, United Kingdom

KEYWORDS chemical vapor deposition, kinetic model, growth mechanism, shape transition,

Kinetic Monte Carlo, $\mathrm{MoS}_{2}, \mathrm{WS}_{2}$, transition metal dichalcogenide monolayer

*Email: strano@mit.edu 


\section{S1. Calculation of Bond Energies in the System}

The Mo-S, Mo-Mo, and S-S bond energies can be computed from the energetics of formation of vacancies in monolayer $\mathrm{MoS}_{2}$. Specifically, if the vacancy formation energies for the Movacancy, the S-vacancy, the MoS-vacancy, and the $\mathrm{MoS}_{2}$-vacancy are available, we can formulate 4 linear equations based on the number of bonds of each type having to be broken, as shown in Table S1.

Table S1. Energy required for the formation of various types of vacancies in $\mathrm{MoS}_{2}$, along with the number of different bonds (Mo-S, Mo-Mo, and S-S) that would need to be broken to create such vacancies.

\begin{tabular}{|l|l|l|l|l|}
\hline Type of Vacancy & $\begin{array}{l}\text { \# of Mo-S } \\
\text { Bonds Bro- }\end{array}$ & $\begin{array}{l}\text { \# of Mo-Mo } \\
\text { Bonds Bro- }\end{array}$ & $\begin{array}{l}\text { \# of S-S } \\
\text { Bonds Bro- }\end{array}$ & $\begin{array}{l}\text { Vacancy For- } \\
\text { mation Energy }\end{array}$ \\
\hline Mo-Vacancy & 6 & ken & ken & $(\mathbf{e V})$ \\
\hline S-Vacancy & 3 & 6 & 0 & 13.44 \\
\hline MoS-Vacancy & 8 & 0 & 7 & 5.89 \\
\hline MoS2-Vacancy & 10 & 6 & 7 & 17.36 \\
\hline
\end{tabular}

These linear equations can be solved using least-squares to obtain:

$E_{M o-S}=1.325 \mathrm{eV}, E_{M o-M o}=0.882 \mathrm{eV}$, and $E_{S-S}=0.288 \mathrm{eV}$ 


\section{S2. Derivation of Linear Scaling between Crystal Size and Growth Time for Regular Hexa- gons and Equilateral Triangles}

The derivation of the linear scaling between crystal size and growth time for truncated triangles was presented as Eqs. (12), (13), and (14) in the main text. Here, we follow a similar procedure for regular hexagons and equilateral triangles.

\section{$\underline{\text { Regular Hexagons }}$}

Consider a crystal growing as a regular hexagon. In this case, as explained in the main text, the growth rates of all the 6 faces of the hexagon will be equal, say $r$. Let $s$ denote the length of each side. If $h$ denotes the height of a monolayer, the volume of the crystal $V$ can be written as follows:

$$
V=\frac{3 \sqrt{3}}{2} s^{2} h
$$

The rate of change of volume with time, $\frac{d V}{d t}$, can be expressed in terms of the growth rate at each of the six faces:

$$
\frac{d V}{d t}=\frac{d}{d t}\left(\frac{3 \sqrt{3}}{2} s^{2} h\right)=r(6 s) h
$$

Equation (S2) is a first-order ordinary differential equation for $s(t)$ which can be solved to yield:

$$
s(t)-s\left(t_{0}\right)=\frac{2 \sqrt{3} r}{3}\left(t-t_{0}\right)
$$

Note that Eq. (S3) corresponds to Eq. (15) in the main text. 


\section{Equilateral Triangles}

Consider a crystal growing as an equilateral triangle. In this case, as explained in the main text, the growth rates of all the 3 faces of the triangle will be equal, say $r$. Let $s$ denote the length of each side. If $h$ denotes the height of a monolayer, the volume of the crystal, $V$, can be written as follows:

$$
V=\frac{\sqrt{3}}{4} s^{2} h
$$

The rate of change of volume with time, $\frac{d V}{d t}$, can be expressed in terms of the growth rate at each of the three faces as follows:

$$
\frac{d V}{d t}=\frac{d}{d t}\left(\frac{\sqrt{3}}{4} s^{2} h\right)=r(3 s) h
$$

Equation (S5) is a first-order ordinary differential equation for $s(t)$ which can be solved to yield:

$$
s(t)-s\left(t_{0}\right)=2 \sqrt{3} r\left(t-t_{0}\right)
$$

Note that Eq. (S6) corresponds to Eq. (16) in the main text. 


\section{S3. Conversion of Experimental Side Lengths to Equivalent Circle Diameters for various}

\section{Crystal Shapes}

The crystal size in the KMC routine is calculated as the diameter of a circle having the same area, $A$, as the crystal (that is, as an equivalent circle diameter). To convert the experimental side lengths into equivalent diameters (for comparison purposes), the following conversion factors are used:

\section{Truncated Triangles}

$$
A=\frac{\pi d^{2}}{4}=\frac{\sqrt{3} s_{1}^{2}}{4}\left[1+4\left(\frac{s_{2}}{s_{1}}\right)+\left(\frac{s_{2}}{s_{1}}\right)^{2}\right] \Rightarrow s_{1}=\left(\frac{\pi}{\sqrt{3}\left[1+4\left(\frac{s_{2}}{s_{1}}\right)+\left(\frac{s_{2}}{s_{1}}\right)^{2}\right]}\right)^{\frac{1}{2}} d
$$

\section{$\underline{\text { Regular Hexagons }}$}

$$
A=\frac{\pi d^{2}}{4}=\frac{3 \sqrt{3}}{2} s^{2} \Rightarrow s=\left(\frac{\pi}{6 \sqrt{3}}\right)^{\frac{1}{2}} d=0.550 d
$$

\section{Equilateral Triangles}

$$
A=\frac{\pi d^{2}}{4}=\frac{\sqrt{3}}{4} s^{2} \Rightarrow s=\left(\frac{\pi}{\sqrt{3}}\right)^{\frac{1}{2}} d=1.347 d
$$

Table S2 below lists the conversion factors for the various crystal shapes at the 5 different locations along the substrate, corresponding to the data of Wang et al. 
Table S2. Conversion of Actual Experimental Crystal Sizes in the Study of Wang et al. to the Corresponding Equivalent Circle Diameters

\begin{tabular}{|l|l|l|l|}
\hline Location & Shape & $\begin{array}{l}\text { Experimental } \\
\text { Side Length, } S\end{array}$ & Equivalent Circle \\
& & $(\mu \mathrm{m})$ & Diameter, $\boldsymbol{d}(\boldsymbol{\mu m})$ \\
\hline$(1)$ & Triangle & 47.9 & 35.6 \\
\hline$(2)$ & Truncated Triangle $(\mathbf{s} 2 / \mathbf{s} 1=0.24)$ & 12.3 & 13.0 \\
\hline$(3)$ & Truncated Triangle $\left(\mathbf{s} 2 / \mathbf{s}_{1}=\mathbf{0 . 3 5}\right)$ & 7.8 & 9.2 \\
\hline$(4)$ & Hexagon & 4.1 & 7.5 \\
\hline$(5)$ & Triangle & 2.8 & 2.1 \\
\hline
\end{tabular}

S4. A Combined Analytical Growth Theory for Triangles, Hexagons, and Truncated Triangles

In order to compare the crystal growth rates of hexagons, triangles, and truncated triangles, we converted the side growth rates to the corresponding rates for the diameter of an equivalent circle. Before deriving the combined analytical growth model for hexagons, triangles, and truncated triangles, we derive the growth equation for a circle.

\section{Growth Equation for a Circle}

As shown earlier for a truncated triangle, a regular hexagon, and an equilateral triangle (see Eqs. (12), (13), (14), (S1), (S2), (S3), (S4), (S5), and (S6)), we can also derive a linear growth rate for the diameter, $d$, of a circular crystal. Specifically, the area $A$ of the crystal and the perimeter $P$ of the crystal are given by: 
$A=\frac{\pi}{4} d^{2}$ and $P=\pi d$, so that:

$$
\frac{d V}{d t}=\frac{d}{d t}\left(\frac{\pi}{4} d^{2} h\right)=r_{\text {circle }}(\pi d) h
$$

Equation (S10) is a first-order ordinary differential equation for $d(t)$ whose solution is given by:

$$
d(t)-d\left(t_{0}\right)=2 r_{\text {circle }}\left(t-t_{0}\right) \equiv k_{\text {circle }}\left(t-t_{0}\right)
$$

where $k_{\text {circle }}$ is an "equivalent circular growth rate constant", and can be readily obtained by fitting KMC simulation data to Eq. (S11). Note that Eq. (S11) corresponds to Eq. (17) in the main text of the paper. Next, we convert the growth rates for various crystal shapes into those for an equivalent circle.

\section{Truncated Triangles}

For truncated triangles, using the conversion factor in Eq. (S7), as well as the relation $r_{1} s_{1}=$ $r_{2} s_{2}$ presented in the main text, in the growth equation Eq. (14) of the main text, yields:

$$
d(t)-d\left(t_{0}\right)=\frac{4 \sqrt{3} r_{1}}{\left(\frac{\pi}{\sqrt{3}}\right)^{1 / 2} \sqrt{1+4\left(\frac{r_{1}}{r_{2}}\right)+\left(\frac{r_{1}}{r_{2}}\right)^{2}}}\left(t-t_{0}\right) \equiv k_{\text {circle }}\left(t-t_{0}\right)
$$

\section{$\underline{\text { Regular Hexagons }}$}

For regular hexagons, using the conversion factor in Eq. (S8) in the growth equation Eq. (S3), yields:

$$
d(t)-d\left(t_{0}\right)=\frac{2 \sqrt{2} r_{\text {face }}}{\left(\frac{\pi}{\sqrt{3}}\right)^{1 / 2}}\left(t-t_{0}\right) \equiv k_{\text {circle }}\left(t-t_{0}\right)
$$

\section{Equilateral Triangles}


For equilateral triangles, using the conversion factor in Eq. (S9) in the growth equation Eq. (S6), yields:

$$
d(t)-d\left(t_{0}\right)=\frac{2 \sqrt{3} r_{\text {face }}}{\left(\frac{\pi}{\sqrt{3}}\right)^{1 / 2}}\left(t-t_{0}\right) \equiv k_{\text {circle }}\left(t-t_{0}\right)
$$

An examination of Eqs. (S12), (S13), and (S14) shows that the growth rates for the three cases considered above can be conveniently written as follows:

$$
\frac{k_{\text {circle }}}{2 \sqrt{3}}\left(\frac{\pi}{\sqrt{3}}\right)^{1 / 2}=\left\{\begin{array}{cc}
r_{1} ; & \text { shape }=\text { triangle } \\
\sqrt{\frac{2}{3} r_{1} ;} & \text { shape }=\text { hexagon } \\
\frac{2 r_{1} r_{2}}{\sqrt{r_{1}^{2}+4 r_{1} r_{2}+r_{2}^{2}}} ; & \text { shape }=\text { truncated triangle }
\end{array}\right.
$$

where faces of type "1", i.e., S-zz faces, dominate $\left(r_{1} \ll r_{2}\right)$. Recognizing that substituting $r_{1}=$ $r_{2}$ in Eq. (S12) yields Eq. (S13), we can rewrite Eq. (S15) as follows:

$$
\frac{k_{\text {circle }}}{2 \sqrt{3}}\left(\frac{\pi}{\sqrt{3}}\right)^{1 / 2}=\left\{\begin{array}{cc}
r_{1} ; & \text { shape }=\text { triangle } \\
\frac{2 r_{1} r_{2}}{\sqrt{r_{1}^{2}+4 r_{1} r_{2}+r_{2}^{2}}} ; & \text { shape }=\text { truncated triangle } / \text { hexagon }
\end{array}\right.
$$

Note that the transition between triangles $\left(r_{1} \ll r_{2}\right)$ and truncated triangles/hexagons $\left(r_{1} \sim r_{2}\right)$ can be assumed to occur at a critical value of $\frac{r_{1}}{r_{2}}=\alpha$, which yields:

$$
\frac{k_{\text {circle }}}{2 \sqrt{3}}\left(\frac{\pi}{\sqrt{3}}\right)^{1 / 2}= \begin{cases}r_{1} ; & \frac{r_{1}}{r_{2}}<\alpha \\ \frac{2 r_{1} r_{2}}{\sqrt{r_{1}^{2}+4 r_{1} r_{2}+r_{2}^{2}}} ; & \frac{r_{1}}{r_{2}} \geq \alpha\end{cases}
$$


Equation (S17) can describe the growth law for equilateral triangles, truncated triangles, and hexagons, when faces of type " 1 " dominate, i.e., for $0 \leq \frac{r_{1}}{r_{2}} \leq 1$. To include cases where faces of type "2" dominate, i.e., $1<\frac{r_{1}}{r_{2}}<\infty$, we recognize that the slower growing face is the dominant one, and modify Eq. (S17) as follows:

$$
\frac{k_{\text {circle }}}{2 \sqrt{3}}\left(\frac{\pi}{\sqrt{3}}\right)^{1 / 2}= \begin{cases}r_{\text {min }} ; & \left(\frac{r_{1}}{r_{2}}\right)_{\min }<\alpha \\ \frac{2 r_{1} r_{2}}{\sqrt{r_{1}^{2}+4 r_{1} r_{2}+r_{2}^{2}}} ; & \left(\frac{r_{1}}{r_{2}}\right)_{\min } \geq \alpha\end{cases}
$$

where $r_{\min }=\min \left(r_{1}, r_{2}\right)$ and $\left(\frac{r_{1}}{r_{2}}\right)_{\min }=\min \left(\frac{r_{1}}{r_{2}}, \frac{r_{2}}{r_{1}}\right)$. Note that Eq. (S18) is identical to the combined analytical growth rate expression, Eq. (18), presented in the main text.

\section{S5. Transport Model}

\section{a. Calculation of Vapor Phase Diffusivities of Precursors}

The diffusivities of the precursors in the carrier gas $(D)$ were calculated using the Chapman-Enskog relation. ${ }^{2}$ Specifically,

$$
D\left[\frac{c m^{2}}{s}\right]=\frac{0.0026280 \sqrt{T^{3}\left(\frac{M_{1}+M_{2}}{2 M_{1} M_{2}}\right)}}{p \sigma_{12}^{2} \Omega_{12}^{(1,1) *}\left(T_{12}^{*}\right)}
$$

where $p$ is the pressure of the system in atm, $T$ is the absolute temperature of the system, $M_{1}$ and $M_{2}$ are the molar masses of the precursor and the carrier gas in $\mathrm{g} / \mathrm{mol}$, respectively, $\left\{\sigma_{i}, \epsilon_{i}\right\}$ are the 12-6 Lennard-Jones parameters of the precursor $(i=1)$ and the carrier gas $(i=2), T_{12}^{*}=\frac{k_{B} T}{\epsilon_{12}}$ is the reduced temperature, and $\Omega_{12}^{(1,1) *}$ is a collision integral. 
Combining rules are applicable such that $\sigma_{12}=\left(\frac{\sigma_{1}+\sigma_{2}}{2}\right)$ and $\epsilon_{12}=\left(\epsilon_{1} \epsilon_{2}\right)^{\frac{1}{2}}$. The values of $\sigma$ and $\epsilon$ are listed in Table $\mathrm{S} 1$; and $\Omega_{12}^{(1,1) *}$ is available in tabular format ${ }^{2}$.

Table S3. 12-6 Lennard-Jones Parameters and Molar Masses of the Various Chemical Species Involved in the CVD Process.

\begin{tabular}{|c|c|c|c|}
\hline & $\boldsymbol{\sigma}(\dot{\mathbf{A}})$ & $\frac{\boldsymbol{\epsilon}}{\boldsymbol{k}_{\boldsymbol{B}}}(\mathbf{K})$ & $\begin{array}{c}\text { Molar Mass } \\
\text { (g/mol) }\end{array}$ \\
\hline Nitrogen $^{2}$ & 3.681 & 91.5 & 28.0 \\
\hline Argon $^{2}$ & 3.418 & 124 & 39.95 \\
\hline Sulfur ${ }^{3}$ & 3.84 & 50 & 32.0 \\
\hline $\mathrm{MoO}_{3}{ }^{3}$ & 4.86 & 100 & 143.94 \\
\hline $\mathrm{WO}_{3}{ }^{3 *}$ & 4.86 & 100 & 231.84 \\
\hline
\end{tabular}

\section{b. Calculation of Carrier Gas Viscosity}

The viscosities of helium and nitrogen (as a function of temperature) were obtained from the National Institute of Standards \& Technology (NIST) online database of fluid properties. ${ }^{4,5}$ For argon, the correlation developed by Younglove and Hanley ${ }^{6}$ (Eq. 1 in ref. 6) was used. 


\section{c. Calculation of Carrier Gas Velocity over Precursor}

The carrier gas velocity was required for use in the mass transfer calculations outlined in Eqs. (9) and (10) of the main text. Calculation of the carrier gas velocity over the precursors can be divided into two cases, as depicted in Figure S1:

i. The precursor is placed in an uncovered boat: flow containing the sublimed precursor occurs over the entire cross-section of the reactor, $A_{r}$.

ii. The precursor is placed in a boat covered on top with substrates: flow containing the sublimed precursor occurs over a reduced cross-section, $A_{\text {boat }}$.

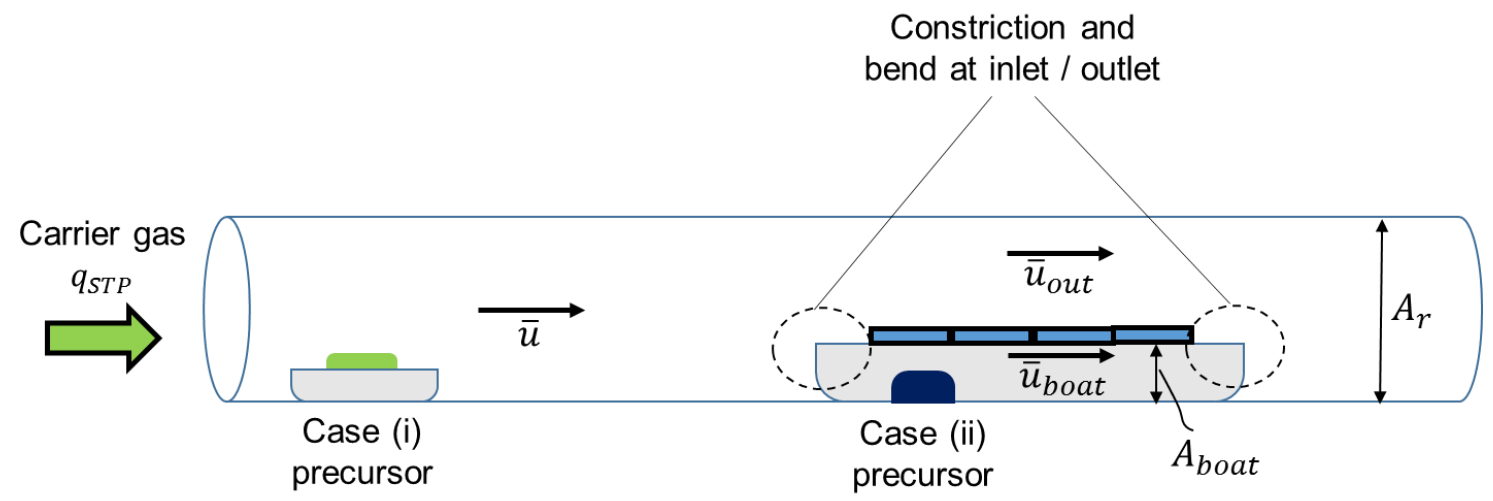

Figure S1. Depiction of carrier gas velocity calculations over various types of precursor arrangements: case (i) where the precursor is in an uncovered boat, and case (ii) where the precursor is in a boat covered on top with substrates.

In case (i), the carrier gas velocity $(\bar{u})$ is just the flow rate of the carrier gas at standard temperature and pressure (STP) conditions $\left(q_{S T P}\right)$, corrected for changes in temperature and pressure, divided by the cross-sectional area of the reactor tube, $A_{r}$, that is,

$$
\bar{u}=\frac{q_{S T P}}{A_{r}}\left(\frac{T[K]}{273.15}\right)\left(\frac{1}{P[a t m]}\right)
$$

In case (ii), the carrier gas velocity in the boat needs to be corrected for the loss in head that happens due to the change in the flow direction. This can be formulated using Ber- 
noulli's equation corrected for head loss. If $\bar{u}=\bar{u}_{\text {boat }}$ is the convective velocity of the gas inside the boat, and $\bar{u}_{\text {out }}$ is the convective velocity around the boat, conservation of mass of the carrier gas requires the following:

$$
\bar{u}_{\text {boat }} A_{\text {boat }}+\bar{u}_{\text {out }}\left(A_{r}-A_{\text {boat }}\right)=q_{\text {STP }}\left(\frac{T[K]}{273.15}\right)\left(\frac{1}{P[a t m]}\right)
$$

Further, the pressure drop encountered by the carrier gas inside the boat must equal the pressure drop in the region outside the boat, so that: $:^{7,8}$

$$
\frac{1}{2} f_{\text {out }} \bar{u}_{\text {out }}^{2}\left(\frac{L_{\text {boat }}}{d_{H, \text { out }}}\right)=\frac{1}{2} f_{\text {boat }} \bar{u}_{\text {boat }}^{2}\left(\frac{L_{\text {boat }}}{d_{H, \text { boat }}}\right)+\frac{1}{2} k_{\text {bends }} \bar{u}_{\text {boat }}^{2}
$$

where $d_{H, \text { out }}$ and $d_{H, \text { boat }}$ are the hydraulic diameters ${ }^{7}$ of the regions around the boat and in the boat, respectively, given by:

$$
d_{H}=\frac{4 \times(\text { Wetted area })}{\text { Perimeter }}
$$

Note that in Eq. (S22), $f$ denotes Darcy's friction factor of the boat / outer region around the boat, given by ${ }^{7}$ :

$$
\begin{aligned}
f_{\text {boat }}=\frac{64}{R e_{\text {boat }}} ; & R e_{\text {boat }}=\frac{\rho \bar{u}_{\text {boat }} d_{H, \text { boat }}}{\mu} \\
f_{\text {out }}=\frac{64}{R e_{\text {out }}} ; & R e_{\text {out }}=\frac{\rho \bar{u}_{\text {out }} d_{H, \text { out }}}{\mu}
\end{aligned}
$$

In addition, in Eq. (S22), $k_{\text {bends }}$ denotes the loss-coefficient due to the two bends $(2 \times$ $0.9)^{8}$ and the two constrictions (area reduction) where the vapor enters the boat $(2 \times 0.5)^{8}$, so that $k_{\text {bends }}=2.8$, and $L_{\text {boat }}$ denotes the length of the boat. 
Equations (S21) and (S22) can be solved simultaneously to yield the value of $\bar{u}_{\text {boat }}$, which can then be used in the transport model. Note that before the mass transfer model can be utilized, one also needs to calculate the saturated vapor phase concentration $\left(C^{*}\right)$, as outlined below.

\section{d. Calculation of Saturated Vapor Phase Concentrations of Precursors}

i. Sulfur: The vapor pressure of sulfur $\left(p^{*}\right)$ is described by the following correlation $(389 \mathrm{~K}<\mathrm{T}<1313 \mathrm{~K})^{9}$ :

$\ln \left(\frac{p^{*}}{p_{c}}\right)=\left[A\left(1-\frac{T}{T_{c}}\right)+B\left(1-\frac{T}{T_{c}}\right)^{\frac{3}{2}}+C\left(1-\frac{T}{T_{c}}\right)^{3}+D\left(1-\frac{T}{T_{c}}\right)^{6}\right]\left(\frac{T_{c}}{T}\right)$

where $T$ is the absolute temperature of the sulfur precursor in Kelvin, $\mathrm{A}=-7.246, \mathrm{~B}$ $=0.187, \mathrm{C}=5.271, \mathrm{D}=-12.128, T_{c}=1313 \mathrm{~K}$, and $p_{c}=18208 \mathrm{kPa}$.

ii. $\mathrm{MoO}_{3}:$ The vapor pressure of $\mathrm{MoO}_{3}\left(p^{*}\right)$ is described by the following correlation $\left(600{ }^{\circ} \mathrm{C}<\mathrm{T}<700{ }^{\circ} \mathrm{C}\right)^{10}$ :

$$
4.576 \log p^{*}\left(\mathrm{MoO}_{3}\right)_{\bar{n}}=-\frac{75400}{T}+62.3 \pm 0.2
$$

where $p^{*}$ is the pressure in atmospheres, $\mathrm{T}$ is the absolute temperature of the $\mathrm{MoO}_{3}$ precursor in Kelvin, and $\bar{n}$ is the average molecular association number. ${ }^{10}$

iii. $\mathrm{WO}_{3}$ : The vapor pressure of $\mathrm{WO}_{3}\left(p^{*}\right)$ is described by the following correlation (800 $\left.{ }^{\circ} \mathrm{C}<\mathrm{T}<1250{ }^{\circ} \mathrm{C}\right)^{11}$

$$
\log p^{*}=17.973-\frac{27295}{T}
$$


where $p^{*}$ is the pressure in $\mathrm{mm} \mathrm{Hg}$, and $\mathrm{T}$ is the absolute temperature of the $\mathrm{WO}_{3}$ precursor in Kelvin

Using the correlations above, one can calculate the required vapor pressures $\left(p^{*}\right)$, which can then be used to calculate the saturated vapor phase molar concentration $\left(C^{*}\right)$, assuming the validity of ideal-gas law for a dilute vapor:

$$
C^{*}(T)=\frac{p^{*}(T)}{R T}
$$

\section{e. Calculation of Actual Vapor Phase Concentrations of Precursors}

The saturated molar concentration $\left(C^{*}\right)$ is then used to calculate the actual concentration $(C)$ in the vapor phase using the molar balance Eq. (10), as outlined in the main text. Note that if the precursor is vaporized at a given temperature $\left(T_{\text {prec }}\right)$ and the substrate is present at a different temperature $\left(T_{\text {subs }}\right)$, the concentration must be corrected for the change in flow rate at the two locations. Specifically,

$$
C_{\text {subs }}=C_{\text {prec }}\left(\frac{T_{\text {prec }}}{T_{\text {subs }}}\right)
$$

\section{S6. Tabulation of Vapor Phase Concentrations in the Various Studies Considered}

In all the studies considered, the growth pressure was $1 \mathrm{~atm}$. The carrier gas in all cases was argon, except for the study of Schmidt et al., where nitrogen was used. The values calculated using our transport model are listed in Table S4 below: 
Table S4. Compilation of the Known and Calculated Process Parameters for the Various Studies Considered to Validate the Model.

\begin{tabular}{|c|c|c|c|c|c|c|c|c|c|c|c|c|c|c|}
\hline Authors & $\begin{array}{l}\text { Gas } \\
\text { Flow } \\
\text { (sccm) }\end{array}$ & $\begin{array}{l}\text { Time } \\
(\mathbf{m i n})\end{array}$ & $\begin{array}{l}\text { d } \\
\text { (") }\end{array}$ & $\begin{array}{l}\mathbf{T}_{\text {Subs }} \\
\left({ }^{\circ} \mathbf{C}\right)\end{array}$ & $\begin{array}{l}\mathbf{u}_{\mathrm{MO3}} \\
(\mathrm{cm} / \mathrm{s})\end{array}$ & $\begin{array}{l}\mathbf{u}_{\mathbf{S}} \\
(\mathbf{c m} / \mathbf{s})\end{array}$ & $\begin{array}{l}\mathbf{T}_{\mathrm{MO3}} \\
\left({ }^{\circ} \mathrm{C}\right)\end{array}$ & $\begin{array}{l}\mathrm{D}_{\mathrm{MO3}} \\
\left(\mathrm{m}^{2} / \mathbf{s}\right)\end{array}$ & $\begin{array}{l}\mathrm{C}_{\text {MO3 }}^{*} \\
(\mathbf{m o l} / \\
\left.\mathbf{m}^{3}\right)\end{array}$ & $\begin{array}{l}\mathrm{C}_{\mathrm{MO3}} \\
(\mathbf{m o l} / \\
\left.\mathbf{m}^{3}\right)\end{array}$ & $\begin{array}{l}\mathbf{T}_{\mathrm{S}} \\
{ }^{\circ} \mathrm{C}\end{array}$ & $\mathrm{D}_{\mathrm{S}}\left(\mathrm{m}^{2} / \mathrm{s}\right)$ & $\begin{array}{l}\mathbf{C}_{\mathrm{s}}^{*} \\
(\mathbf{m o l} / \\
\left.\mathbf{m}^{3}\right)\end{array}$ & $\begin{array}{l}\mathrm{C}_{\mathrm{S}} \\
(\mathbf{m o l} / \\
\left.\mathbf{m}^{3}\right)\end{array}$ \\
\hline
\end{tabular}

\begin{tabular}{|c|c|c|c|c|c|c|c|c|c|c|c|c|c|c|}
\hline \multicolumn{15}{|l|}{$\mathbf{M o S}_{2}$} \\
\hline $\begin{array}{l}\text { Wang et } \\
\text { al. }{ }^{12}\end{array}$ & 10 & 10 & 1 & 700 & 0.063 & 0.051 & 700 & $\begin{array}{r}7.76 \mathrm{E}- \\
05\end{array}$ & 0.0207 & 0.0159 & 150 & $3.5 \mathrm{E}-05$ & 0.0073 & $9.65 \mathrm{E}-04$ \\
\hline Lee et al. ${ }^{13}$ & 10 & 5 & 1 & 650 & 0.042 & 0.055 & 650 & $\begin{array}{r}7.1 \mathrm{E}- \\
05\end{array}$ & 0.0026 & 0.0023 & 180 & $3.94 \mathrm{E}-05$ & 0.0314 & $\begin{array}{r}4.762 \mathrm{E}- \\
03\end{array}$ \\
\hline $\begin{array}{l}\text { Schmidt } e t \\
\text { al. }^{14}\end{array}$ & 30 & 5 & 2 & 700 & 0.006 & 0.041 & 700 & $\begin{array}{r}8.63 \mathrm{E}- \\
05\end{array}$ & 0.0207 & 0.0206 & 180 & $4.12 \mathrm{E}-05$ & 0.0314 & $1.5 \mathrm{E}-03$ \\
\hline
\end{tabular}

\section{$\mathrm{WS}_{2}$}

\begin{tabular}{|c|c|c|c|c|c|c|c|c|c|c|c|c|c|c|}
\hline Lee et $a ._{.}{ }^{13}$ & 5 & 5 & 1 & 800 & 0.024 & $\begin{array}{r}0.027 \\
3\end{array}$ & 800 & $\begin{array}{r}8.75 \mathrm{E}- \\
05\end{array}$ & $\begin{array}{r}5.16 \mathrm{E}- \\
10\end{array}$ & $\begin{array}{r}4.93 \mathrm{E}- \\
10\end{array}$ & 180 & $3.94 \mathrm{E}-05$ & 0.0314 & $\begin{array}{r}5.397 \mathrm{E}- \\
03\end{array}$ \\
\hline $\begin{array}{l}\text { Rong et } \\
\text { al. } .^{15}\end{array}$ & 100 & 60 & 1 & 860 & 1.617 & 0.546 & 1070 & $\begin{array}{r}0.0001 \\
26\end{array}$ & $\begin{array}{r}5.35 \mathrm{E}- \\
05\end{array}$ & $\begin{array}{r}6.44 \mathrm{E}- \\
06\end{array}$ & 180 & $3.94 \mathrm{E}-05$ & 0.0314 & $\begin{array}{r}1.385 \mathrm{E}- \\
03\end{array}$ \\
\hline $\begin{array}{l}\text { Peimyoo et } \\
\text { al. }{ }^{16}\end{array}$ & 200 & 10 & 2 & 800 & 0.646 & 0.315 & 800 & $\begin{array}{r}8.75 \mathrm{E}- \\
05\end{array}$ & $\begin{array}{r}5.16 \mathrm{E}- \\
10\end{array}$ & $\begin{array}{r}1.81 \mathrm{E}- \\
11\end{array}$ & 250 & $5.02 \mathrm{E}-05$ & 0.3893 & $\begin{array}{r}8.046 \mathrm{E}- \\
03\end{array}$ \\
\hline
\end{tabular}

Notation: $d$ denotes the diameter of the reactor tube, $\mathrm{T}_{\text {Subs }}$ denotes the temperature of the substrate, subscript $\mathrm{MO}_{3}$ denotes the dichalcogenide precursor, subscript $\mathrm{S}$ denotes the sulfur precursor, $\mathrm{u}$ denotes the carrier gas velocity over the respective precursor, $\mathrm{T}$ denotes the temperature of the respective precursor, $\mathrm{D}$ denotes the vapor phase diffusivity of the respective precursor, $\mathrm{C}^{*}$ denotes the saturated vapor phase concentration of the respective precursor, and $\mathrm{C}$ denotes the actual vapor phase concentration of the respective precursor. 


\section{S7. Linear Trends of Simulated Crystal Sizes}

The data for the crystal size (equivalent circle diameter), $d(t)$, versus time $t$ obtained from the KMC simulations was fit to Eq. (S11) to obtain the "equivalent circular growth rate constant", $k_{\text {circle }}$. The fits for three different cases of $\mathrm{MoS}_{2}$ growth at locations (1)-(4) of the Wang et al. ${ }^{12}$ reactor are shown in Figure S2 below. In all four cases, the data conforms really well to the linear fit.
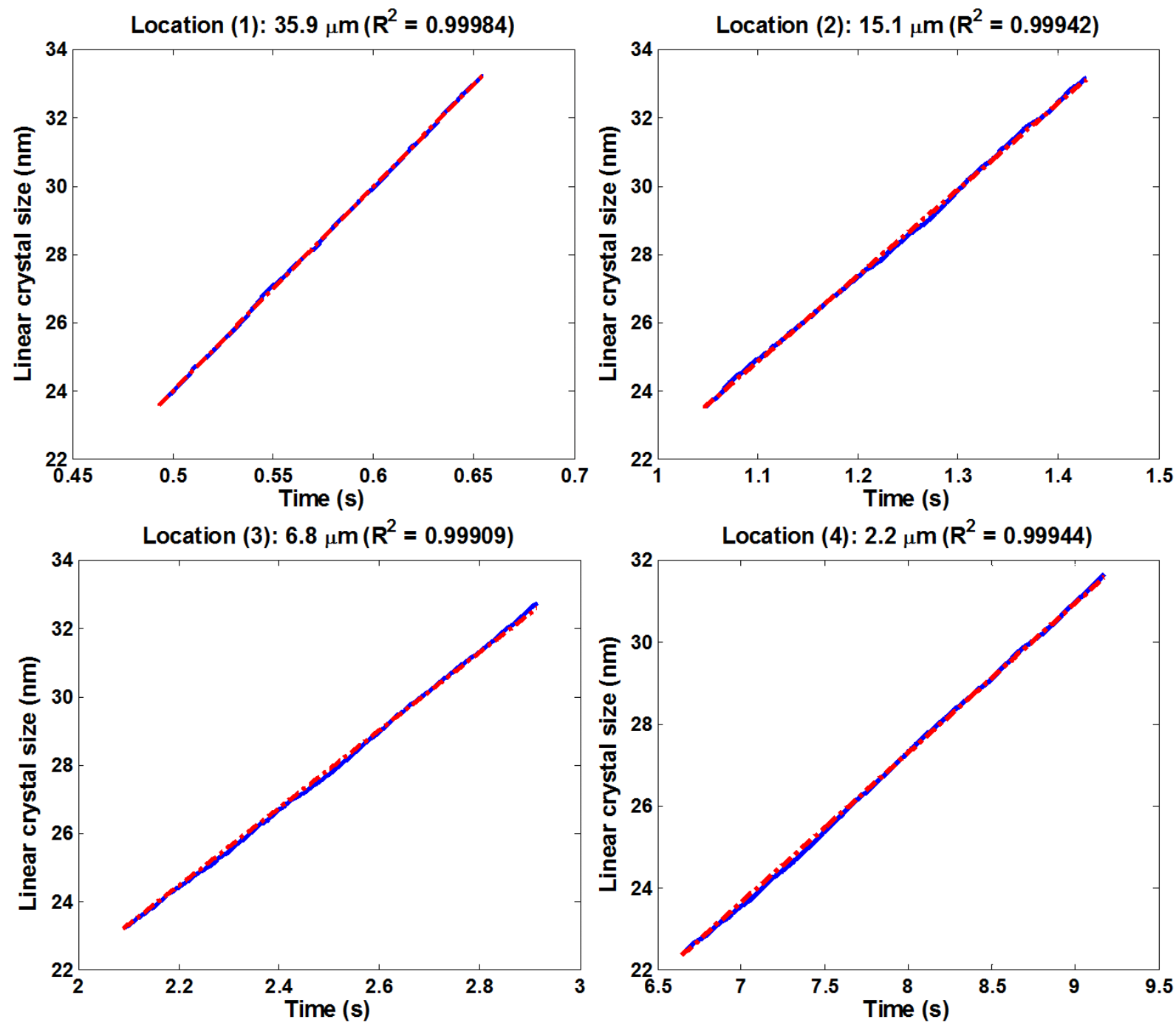

Figure S2. Linear trends for the size of MoS2 crystals at different locations (1), (2), (3) and (4) in the Wang et al. ${ }^{12}$ reactor for a single KMC simulation using the calculated concentra- 
tion profile. Size data was collected after 10,000 KMC time steps to allow the crystal shape to attain steady state, and was taken up to $20,000 \mathrm{KMC}$ time steps. The final extrapolated equivalent circle diameter at 10 minutes, and the $\mathbf{R}^{2}$ value of the fit are listed in parenthesis adjacent to the heading for each plot. The simulated KMC values are shown in blue color, and the linear fit is shown in dashed red color. 
S8. "Kinetic Phase Diagram" for $\mathrm{MoS}_{2}$ CVD Growth at Various Temperatures

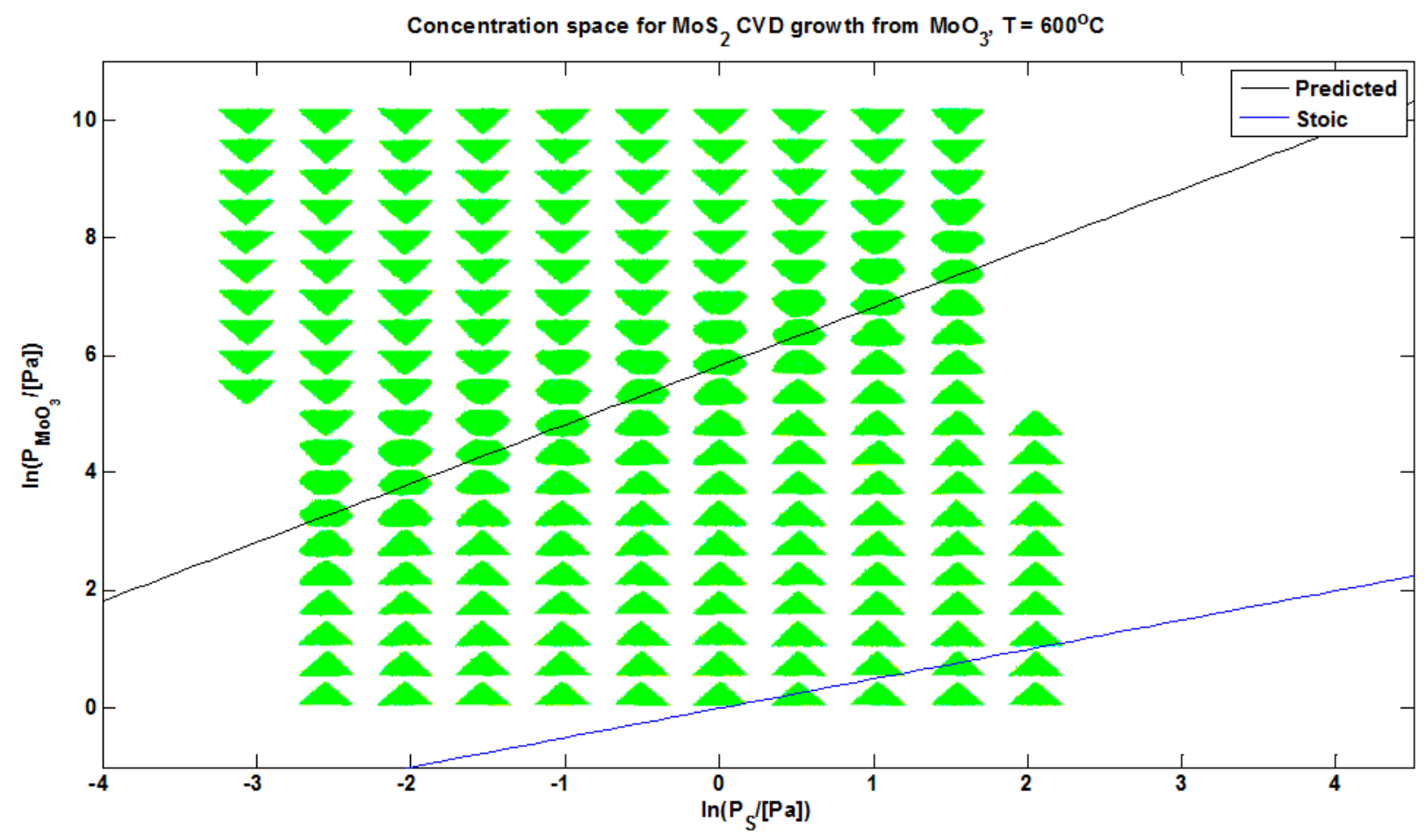

Figure S3. Crystal shapes obtained from the KMC simulation at a temperature of $600^{\circ} \mathrm{C}$. The black line corresponds to Eq. (23) in the main text, and the blue line is the stoichiometric 2:1 S:Mo line. Note that the black line shifts to the left as the temperature decreases from $700{ }^{\circ} \mathrm{C}$, and that all the simulated points shown in the Figure correspond to less than $5 \%$ desorption of atoms (i.e., <1000 steps out of 20,000 KMC time steps), so that Eqs. (18)(20) of the main text, which require the growth to be irreversible, are applicable. The S:Mo ratio in the top-leftmost shape is 1.962 , whereas that in the bottom-rightmost shape is 2.035, with other crystals having values in between. This confirms that no undesirable compounds are formed in the growth process. 


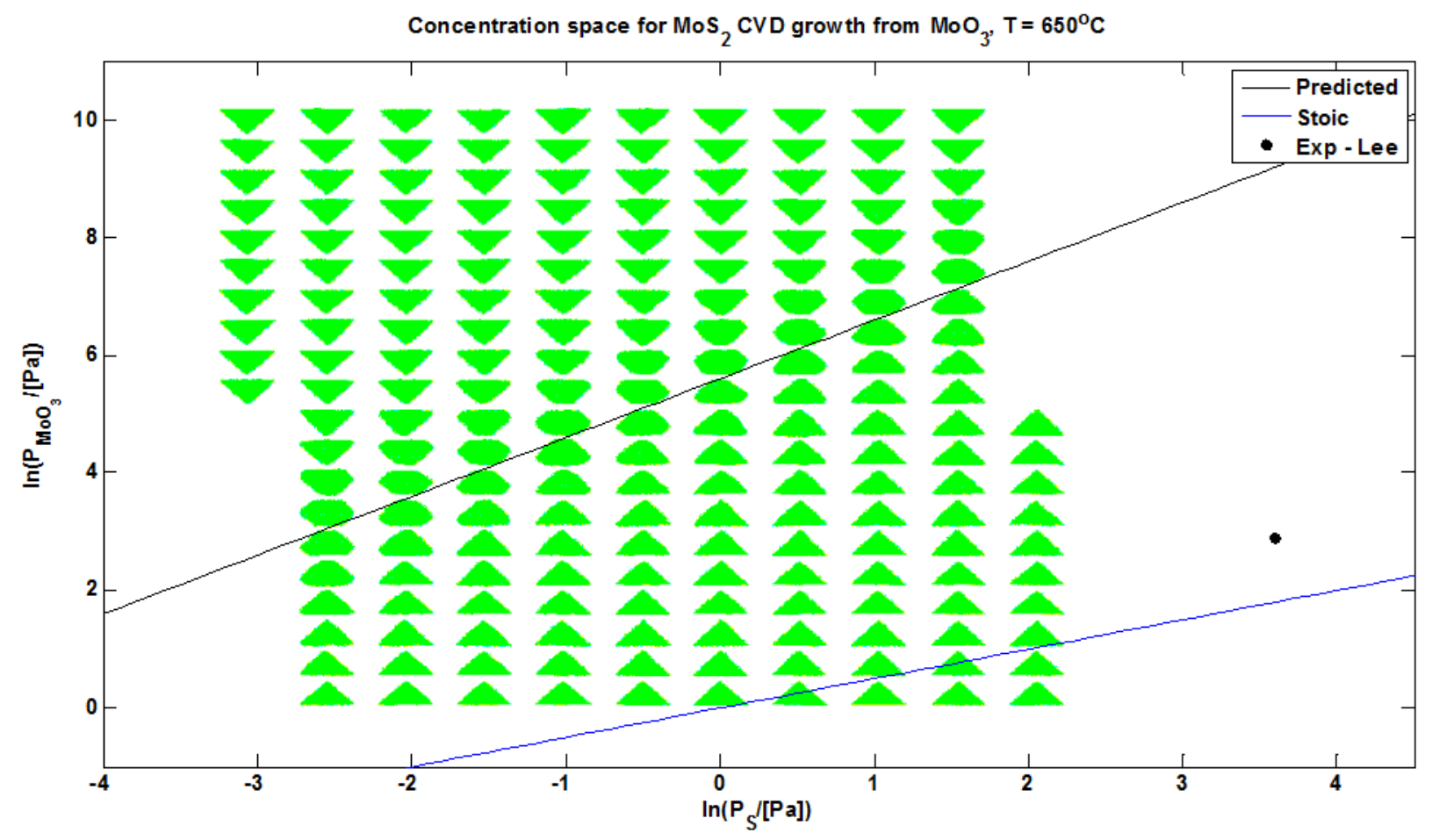

Figure S4. Crystal shapes obtained from the KMC simulation at a temperature of $650{ }^{\circ} \mathrm{C}$. The black line corresponds to Eq. (23) in the main text, and the blue line is the stoichiometric 2:1 S:Mo line. Note that the black line shifts to the left as the temperature decreases from $700{ }^{\circ} \mathrm{C}$, and that all the simulated points shown in the Figure correspond to less than $5 \%$ desorption of atoms (i.e., <1000 steps out of $20,000 \mathrm{KMC}$ time steps), so that Eqs. (18)(20) of the main text, which require the growth to be irreversible, are applicable. The S:Mo ratio in the top-leftmost shape is 1.959 , whereas that in the bottom-rightmost shape is 2.035, with other crystals having values in between. This confirms that no undesirable compounds are formed in the growth process. 


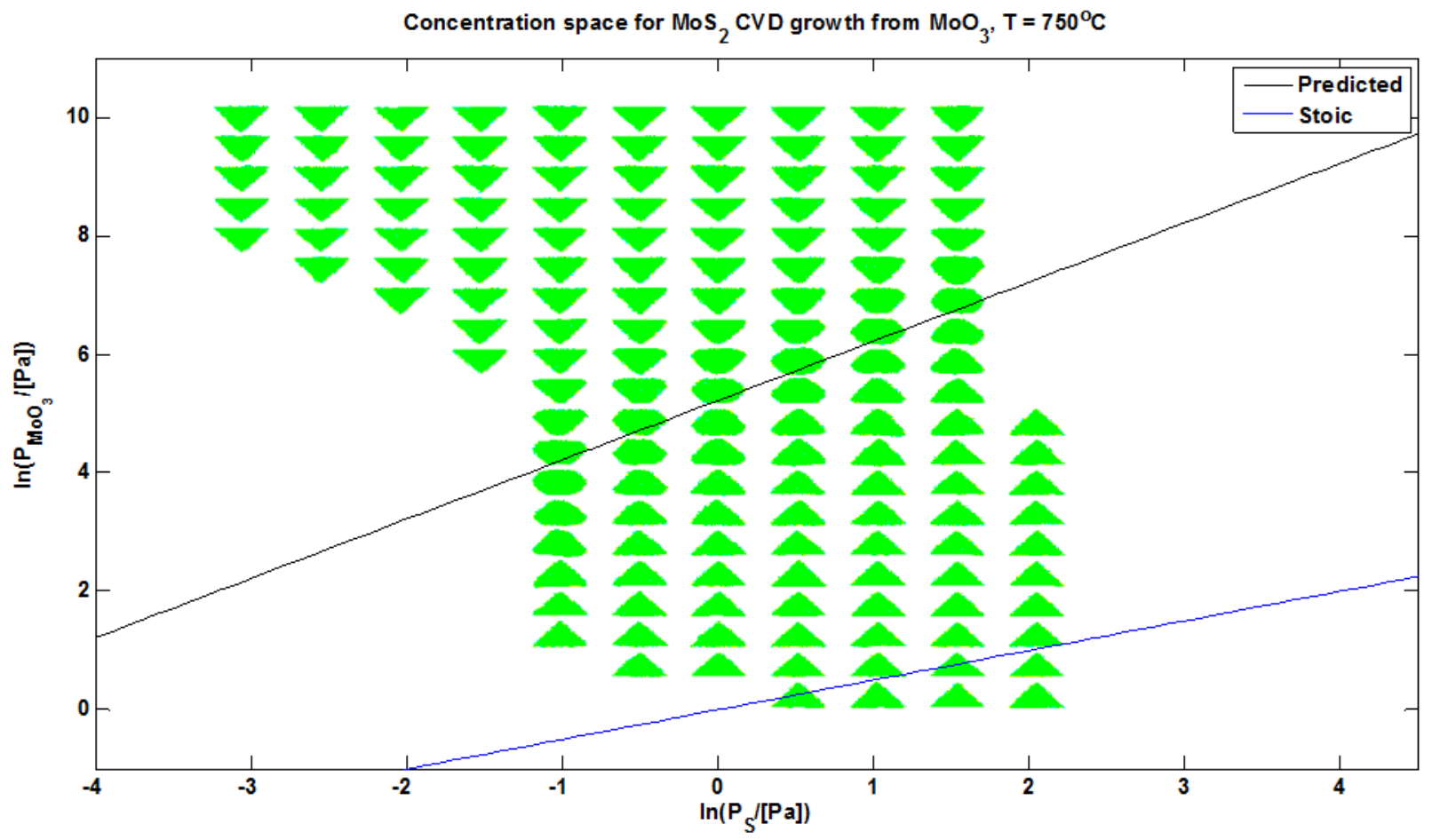

Figure S5. Crystal shapes obtained from the KMC simulation at a temperature of $750{ }^{\circ} \mathrm{C}$. The black line corresponds to Eq. (23) in the main text while the blue line is the stoichiometric 2:1 S:Mo line. Note that the black line moves to the right as the temperature increases from $700{ }^{\circ} \mathrm{C}$. Only points which had less than $5 \%$ desorption of atoms (i.e., $<1000$ steps out of 20,000 KMC time steps) are shown here because the theory (Eqs. (18)-(20) in the main text) is only valid for irreversible growth. The S:Mo ratio in the top-leftmost shape is $\mathbf{1 . 9 5 5}$, whereas that in the bottom-rightmost shape is $\mathbf{2 . 0 3 6}$, with other crystals having values in between. This confirms that no undesirable compounds are formed in the growth process. 
S9. Scanning Electron Microscopy (SEM) Images of MoS $\mathrm{M}_{2}$ Crystals from the Wang et al. Study.

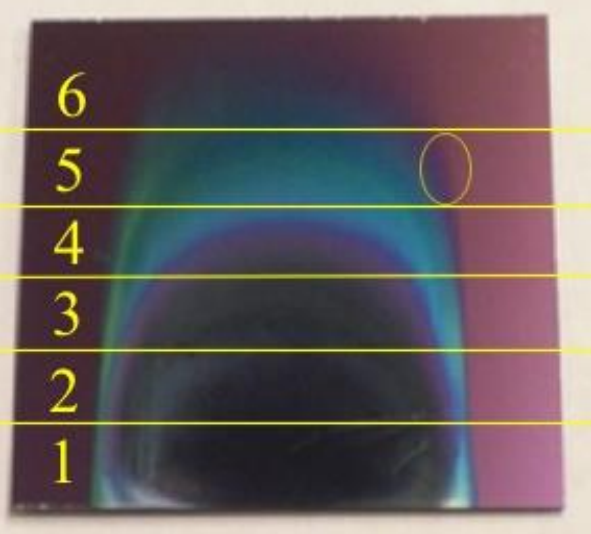

Figure S6. Optical photo showing 6 different regions on the $\mathrm{SiO}_{2}$ substrate from the Wang et al. study. 


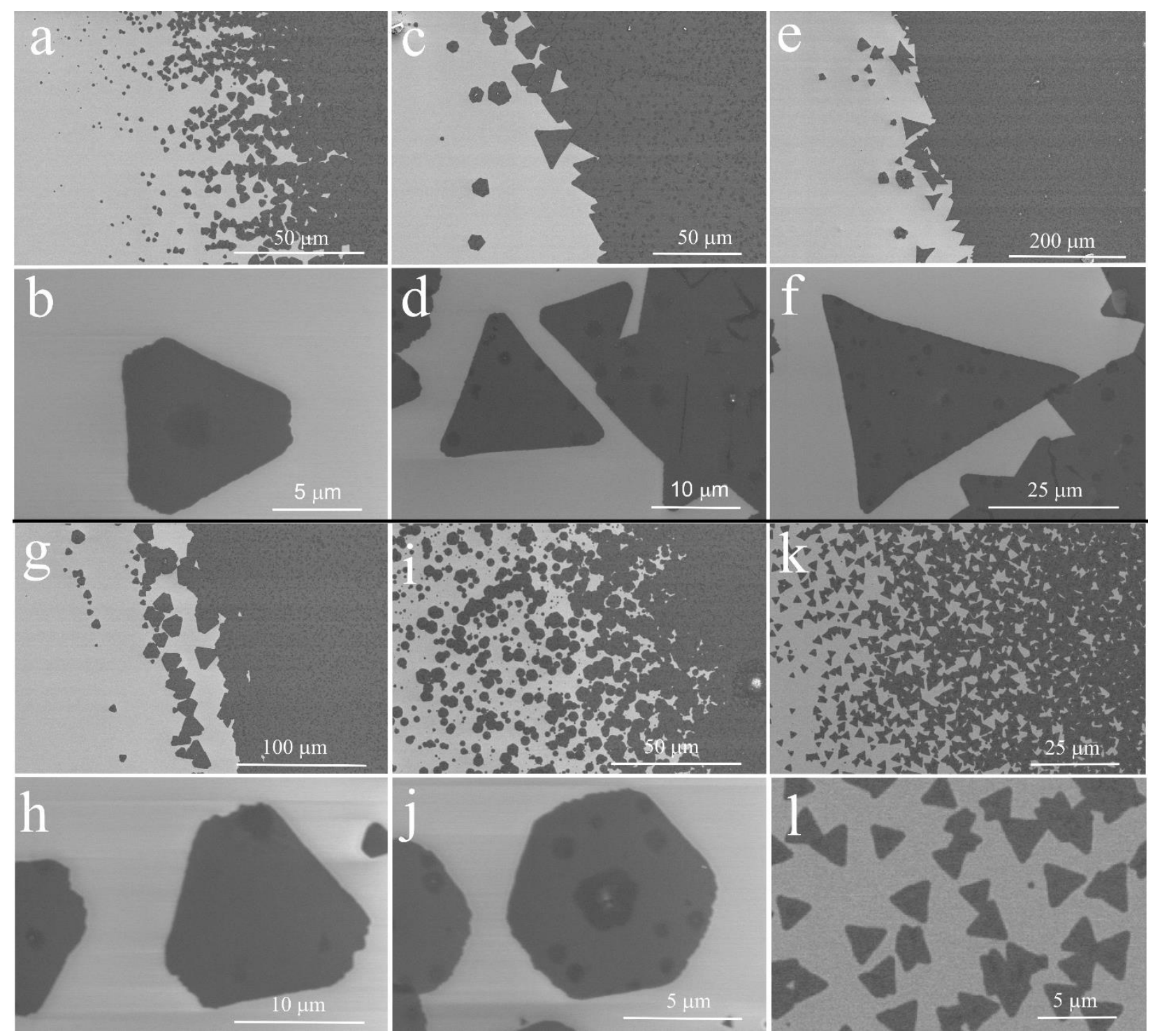

Figure S7. Low magnification and high magnification Scanning Electron Microscopy (SEM) images from each of the 6 regions reported in the Wang et al. study and depicted in Figure S6. (a-b) Region 1, (c-d) Region 2, (e-f) Region 3, (g-h) Region 4, (i-j) Region 5, and (k-l) Region 6.

\section{S10. Note on the Mechanism of $\mathrm{MoS}_{2}$ CVD Growth}

Instead of Mo and S atoms getting attached to the growing lattice one-by-one, another plausible mechanism is where $\mathrm{MoS}_{2}$ units are formed in the vapor phase, and then get attached to the growing lattice. However, in such a scenario, there would be no shape transition, because then, it 
would always be the same concentration of incoming $\mathrm{MoS}_{2}$ groups for the S-zz and Mo-zz faces. In other words, the only difference between the S-zz and the Mo-zz edges would be their different lattice environments, which would not change with location in the reactor, even if there were a concentration gradient. The experimental data of Wang et al. ${ }^{12}$ clearly rule out this alternate mechanism. Note that so far there has been no experimental study on the vapor phase characterization of the intermediates in the process, although there have been studies directed at the solid phase chemistry. ${ }^{17}$

\section{S11. Comparison of the Model when Employing 6 and 7 Next-Nearest Neighbors}

Table S5. The crystal sizes and S:Mo ratios obtained at locations (1-4) of the Wang et al. reactor, using the KMC model, when employing (i) 6 next-nearest neighbors, and (ii) 7 next-nearest neighbors for $\alpha / \gamma$ sites in the model.

\begin{tabular}{|c|l|l|l|l|}
\hline Location in & \multicolumn{2}{|c|}{ (i) Next-Nearest Neighbors } & \multicolumn{2}{|c|}{ (ii) 7 Next-Nearest Neighbors } \\
\hline 1 & 34.12 & S:Mo Ratio & Crystal Size $(\mu \mathrm{m})$ & S:Mo Ratio \\
\cline { 2 - 5 } & Crystal Size $(\mu \mathrm{m})$ & S:M27 & 35.24 & 2.028 \\
\hline 2 & 14.44 & 2.009 & 15.26 & 2.012 \\
\hline 3 & 5.93 & 1.986 & 6.90 & 1.989 \\
\hline 4 & 1.88 & 1.972 & 2.25 & 1.973 \\
\hline
\end{tabular}




\section{References}

(1) Ataca, C.; Ciraci, S. Functionalization of Single-Layer $\mathrm{MoS}_{2}$ Honeycomb Structures. $J$. Phys. Chem. C 2011, 115, 13303-13311.

(2) Hirschfelder, J. O.; Curtiss, C. F.; Bird, R. B.; others. Molecular Theory of Gases and Liquids; Wiley New York, 1954; Vol. 26.

(3) McGee, B. C., M. L. Hobbs, and M. R. B. Exponential 6 Parameterization for the JCZ3EOS.; 1998.

(4) Thermophysical Properties of Helium http://webbook.nist.gov/cgi/fluid.cgi?ID=C7440597\&Action=Page.

Thermophysical Properties of Nitrogen http://webbook.nist.gov/cgi/fluid.cgi?ID=C7727379\&Action=Page.

(6) Younglove, B. A.; Hanley, H. J. M. The Viscosity and Thermal Conductivity Coefficients of Gaseous and Liquid Argon. J. Phys. Chem. Ref. Data 1986, 15, 1323.

(7) Incropera, F. P.; Dewitt, D. P.; Bergman, T. L.; Lavine, A. S. Fundamentals of Heat and Mass Transfer; Sixth Edit.; John Wiley \& Sons, Inc., 2007.

(8) Shames, I. H. Mechanics of Fluids; Sixth Edit.; McGraw Hill, 2003.

(9) Peng, D.-Y.; Zhao, J. Representation of the Vapour Pressures of Sulfur. J. Chem. Thermodyn. 2001, 33, 1121-1131.

(10) Gulbransen, E. A.; Andrew, K. F.; Brassart, F. A. Vapor Pressure of Molybdenum Trioxide. J. Electrochem. Soc. 1963, 110, 242.

(11) Meyer, G.; Oosterom, J. F.; de Roo, J. L. The Vapour Pressure of Tungsten Trioxide. Recl. des Trav. Chim. des Pays-Bas 1959, 78, 412-416.

(12) Wang, S.; Rong, Y.; Fan, Y.; Pacios, M.; Bhaskaran, H.; He, K.; Warner, J. H. Shape Evolution of Monolayer $\mathrm{MoS}_{2}$ Crystals Grown by Chemical Vapor Deposition. Chem. Mater. 2014, 26, 6371-6379. 
(13) Lee, Y.-H.; Yu, L.; Wang, H.; Fang, W.; Ling, X.; Shi, Y.; Lin, C.-T.; Huang, J.-K.; Chang, M.-T.; Chang, C.-S.; Dresselhaus, M.; Palacios, T.; Li, L.-J.; Kong, J. Synthesis and Transfer of Single-Layer Transition Metal Disulfides on Diverse Surfaces. Nano Lett. 2013, 13, 1852-1857.

(14) Schmidt, H.; Wang, S.; Chu, L.; Toh, M.; Kumar, R.; Zhao, W.; Castro Neto, A. H.; Martin, J.; Adam, S.; Özyilmaz, B.; Eda, G. Transport Properties of Monolayer $\mathrm{MoS}_{2}$ Grown by Chemical Vapor Deposition. Nano Lett. 2014, 14, 1909-1913.

(15) Rong, Y.; Fan, Y.; Leen Koh, A.; Robertson, A. W.; He, K.; Wang, S.; Tan, H.; Sinclair, R.; Warner, J. H. Controlling Sulphur Precursor Addition for Large Single Crystal Domains of $\mathrm{WS}_{2}$. Nanoscale 2014, 6, 12096-12103.

(16) Peimyoo, N.; Shang, J.; Cong, C.; Shen, X.; Wu, X.; Yeow, E. K. L.; Yu, T. Nonblinking, Intense Two-Dimensional Light Emitter: Monolayer $\mathrm{WS}_{2}$ Triangles. ACS Nano 2013, 7 , 10985-10994.

(17) Weber, T.; Muijsers, J. C.; van Wolput, J. H. M. C.; Verhagen, C. P. J.; Niemantsverdriet, J. W. Basic Reaction Steps in the Sulfidation of Crystalline $\mathrm{MoO}_{3}$ to $\mathrm{MoS}_{2}$, As Studied by X-Ray Photoelectron and Infrared Emission Spectroscopy. J. Phys. Chem. 1996, 100, 14144-14150. 